\title{
RICCI SOLITONS ON HOPH HYPERSURFACES IN A SASAKIAN SPACE FORM
}

\author{
Zahra Nazari and Esmail Abedi
}

\begin{abstract}
We are studying Ricci solitons on Hoph hypersurfaces in a Sasakian space form $\widetilde{M}^{2 n+1}(c)$. First, we prove that Hoph hypersurfaces of a Sasakian space form $\widetilde{M}^{2 n+1}(c<1)$ with two distinct principal curvatures are shrinking, and for $c \geq 1$ Hoph hypersurfaces with two distinct principal curvatures of a Sasakian space form $\widetilde{M}^{2 n+1}(c)$ do not admit the Ricci soliton. We show that there are no Hoph hypersurfaces with two distinct principal curvatures in a Sasakian space form $\widetilde{M}^{2 n+1}(c)$ with an $\eta$-Ricci soliton (and a Ricci soliton) such that a potential vector field is the Reeb vector field. Then we prove that Hoph hypersurfaces in a Sasakian space form $\widetilde{M}^{2 n+1}(c)$ with $c=1$ do not admit an $\eta$ - Ricci soliton with a potential vector field $U$ and we show that the Ricci soliton on Hoph hypersurfaces $M$ in a Sasakian space form $\widetilde{M}^{2 n+1}(c<-3)$ with a potential vector field $U$ is shrinking the Ricci soliton. Finally, we study the Ricci soliton on locally symmetric hypersurfaces in a Sasakian space form $\widetilde{M}^{2 n+1}(c)$ and prove that the Ricci soliton shrins for $c<1$. Also, there are no locally symmetric hypersurfaces $(M, g)$ of a Sasakian space form $\widetilde{M}^{2 n+1}(c \geq 1)$ with a Ricci soliton.
\end{abstract}

Keywords: Ricci soliton, $\eta$-Ricci soliton, Sasakian space form, locally symmetric hypersurfaces

\section{Introduction}

A smooth vector field $V$ on a Riemannian manifold $(M, g)$ is said to be a Ricci soliton if it satisfies

$$
\frac{1}{2} \mathcal{L}_{V} g+R i c-\lambda g=0
$$

where $L_{V} g$ denotes the Lie-derivative of the metric $g$ with respect to $V$, Ric is the Ricci tensor of $(M, g)$, and $\lambda$ is a constant. We shall show a Ricci soliton by $(M, g, V, \lambda)$ the vector field $V$ of which is a potential vector field of the Ricci soliton. A Ricci soliton $(M, g, V, \lambda)$ is called expanding, steady and shrinking according to

Received June 21, 2016; accepted August 06, 2016

2010 Mathematics Subject Classification. Primary 53C25, 53C15 
$\lambda<0, \lambda=0$ and $\lambda>0$, respectively.

Compact Ricci solitons are fixed points of the Ricci flow:

$$
\frac{\partial}{\partial t} g(t)=-2 \operatorname{Ric}(g(t))
$$

projected from the space of metric tensors onto its quotient modulo diffeomorphisms and scalings, and mostly arise as blow-up limits for the Ricci flow on compact Riemannian manifolds. Furthermore, Ricci solitons model the formation of singularities in the Ricci flow and they correspond to self-similar solutions[11]. Naturally, a trivial Ricci soliton is an Einstein metric with $V$ Killing or zero. A Ricci soliton $(M, g, V ; \lambda)$ is called a gradient Ricci soliton if its potential vector field $V$ is the gradient of the smooth function $f$ on $M$. We denote gradient Ricci soliton by $(M, g, V ; \lambda)$ and call the smooth function $f$ a potential function. Ivey [8] and Hamilton [6] proved that a Ricci soliton on a compact Riemannian manifold in dimension 2 and 3 has a constant curvature. Perelman [12] proved that the potential vector field in a compact Ricci soltion is the sum of a gradient and a Killing vector field. Thus compact Ricci solitons are gradient Ricci solitons. In particular, since Perelman used Ricci solitons it has become more important to solve the long standing Poincaré conjecture discussed in 1904. In [5], Chow and Knopf studied details about gradient Ricci solitons or Ricci solitons.

In [9], Ki proved that there are no real hypersurfaces with a parallel Ricci tensor in a complex space form $\widetilde{M}^{n}(c)$ with $c \neq 0$ when $n \geq 3$. Kim [10] proved that when $n=2$, this is also true. In particular, these results show that there are no Einstein real hypersurfaces in a non-flat complex space form.

In [2], Chen studied the important results on Ricci solitons which obviously occur on some Riemannian submanifolds. He presented several recent new criteria for trivial compact shrinking Ricci solitons.

Cho and Kimura [3] studied Ricci solitons of real hypersurfaces in a non-flat complex space form and showed that a real hypersurface $M$ in a non-flat complex space form $\widetilde{M}^{n}(c \neq 0)$ does not admit a Ricci soliton such that the Reeb vector field $\xi$ is a potential vector field. They defined the so-called $\eta$-Ricci soliton, such that it satisfies

$$
\frac{1}{2} \mathcal{L}_{V} g+R i c-\lambda g-\mu \eta \otimes \eta=0
$$

where $\lambda, \mu$ are constants. They first proved that a real hypersurface $M$ of a non-flat complex space form $\widetilde{M}^{n}(c)$ which accepts an $\eta$-Ricci soliton is a Hopf-hypersurface and classified the $\eta$-Ricci soliton real hypersurfaces in a non-flat complex space form.

Since Ricci solitons are generalization of Einstein manifolds, one may look for new examples in the class where there are Einstein manifolds. A wealthy class of Einstein manifolds has been found in the class of Sasakian manifolds. In [1], 
we can see interesting examples of Sasakian-Einstein manifolds consistuting exotic spheres. Note that all examples of shrinking Ricci solitons are Kähler so far and of even dimensions, whereas Sasakian manifolds are of odd dimension. In addition, a class of examples of Sasakian-Einstein metric is created on $S^{1}$ - bundles over KählerEinstein manifolds. He and Zhu [7], showed that a Sasakian metric which so satisfies the gradient Ricci soliton equation is presently Einstein.

Our paper is structured as follows. The first section is a very brief review of Sasakian space forms and Hoph hypersurfaces, $C R$ hypersurfaces and locally symmetric hypersurfaces in Sasakian space forms and Ricci solitons. In what follows, we study Ricci solitons on Hoph hypersurfaces in Sasakian a space form $\widetilde{M}^{2 n+1}(c)$. Firstly, we prove that Hoph hypersurfaces of a Sasakian space form $\widetilde{M}^{2 n+1}(c<1)$ with two distinct principal curvatures are shrinking and for $c \geq 1$ Hoph hypersurfaces with two distinct principal curvatures of a Sasakian space form $\widetilde{M}^{2 n+1}(c)$ do not admit a Ricci soliton. We show that there are no Hoph hypersurfaces with two distinct principal curvatures in a Sasakian space form $\widetilde{M}^{2 n+1}(c)$ with an $\eta$-Ricci soliton (and a Ricci soliton) such that the potential vector field is the Reeb vector field.

Then we prove that Hoph hypersurfaces in a Sasakian space form $\widetilde{M}^{2 n+1}(c)$ with $c=1$ do not admit an $\eta$ - Ricci soliton with a potential vector field $U$ and we show that the Ricci soliton on Hoph hypersurfaces $M$ in a Sasakian space form $\widetilde{M}^{2 n+1}(c<-3)$ with a potential vector field $U$ is a shrinking Ricci soliton. Finally, we study the Ricci soliton on locally symmetric hypersurfaces in a Sasakian space form $\widetilde{M}^{2 n+1}(c)$ and prove that the Ricci soliton is shrinking for $c<1$. Also, there are not any locally symmetric hypersurfaces $(M, g)$ of a Sasakian space form $\widetilde{M}^{2 n+1}(c \geq 1)$ with a Ricci soliton.

\subsection{PRELIMINARIES}

Definition 1.1. Let $M^{2 n+1}$ be an odd-dimensional manifold. An almost contact structure $(\varphi, \eta, \xi)$ on a differentiable manifold $M^{2 n+1}$ is a vector field $\xi$, a one-form $\eta$ and a (1,1)-tensor field $\varphi$ which satisfy

$$
\varphi^{2}=-I d+\eta \otimes \xi, \quad \eta(\xi)=1, \quad \varphi \xi=0, \quad \eta \circ \varphi=0 .
$$

Then $\left(M^{2 n+1}, \varphi, \eta, \xi\right)$ is an almost contact manifold.

Definition 1.2. If an almost contact manifold $M^{2 n+1}$ admits a Riemannian metric compatible $g$ such that

$$
g(\varphi X, \varphi Y)=g(X, Y)-\eta(X) \eta(Y), \quad \eta(X)=g(X, \xi),
$$

then $\left(M^{2 n+1}, g, \varphi, \eta, \xi\right)$ is an almost contact metric manifold. 
Definition 1.3. A manifold $M^{2 n+1}$ is said to be a contact manifold if it carries a global one-form $\eta$ such that

$$
\eta \wedge(d \eta)^{n} \neq 0
$$

everywhere on $M^{2 n+1}$ and one-form $\eta$ is called the contact form.

Definition 1.4. An almost contact metric manifold $M^{2 n+1}$ is said to be a Sasakian manifold with an almost contact metric structure $(\varphi, \xi, \eta, g)$ if

$$
\begin{aligned}
& \left(\nabla_{X} \varphi\right) Y=g(X, Y) \xi-\eta(Y) X \quad \forall X, Y \in T M \\
& \nabla_{X} \xi=-\varphi X
\end{aligned}
$$

such that, $\nabla$ denotes its Levi-Civita connection of the Riemannian metric $g$.

Definition 1.5. A plane section $\sigma \subset T_{p}(M)$ of a Sasakian manifold $M^{2 n+1}$ is called a $\varphi$-section if $\sigma \perp \xi$ and $\varphi(\sigma)=\sigma$. The restriction sectional curvature $k$ to $\varphi$ section of the Riemannian sectional curvature (of $(M, g)$ ) is the $\varphi$-section curvature. A Sasakian space form is a Sasakian manifold with a constant $\varphi$-sectional curvature.

In this case, the Riemannian curvature tensor field $R$ of a Sasakian space form $M^{2 n+1}(c)$ is given by

$$
\begin{aligned}
R(X, Y) Z & =\frac{c+3}{4}\{g(Y, Z) X-g(X, Z) Y\} \\
& -\frac{c-1}{4}\{\eta(Z)[\eta(Y) X-\eta(X) Y]+[g(Y, Z) \eta(X)-g(X, Z) \eta(Y)] \xi \\
& -g(\varphi Y, Z) \varphi X+g(\varphi X, Z) \varphi Y+2 g(\varphi X, Y) \varphi Z\} .
\end{aligned}
$$

Definition 1.6. A smooth vector field $V$ on a Riemannian manifold $(M, g)$ is said to be a Ricci soliton if it satisfies

$$
\frac{1}{2} \mathcal{L}_{V} g+R i c-\lambda g=0
$$

where $L_{V} g$ denotes the Lie-derivative of the metric $g$ with respect to $V$, Ric is the Ricci tensor of $(M, g)$ and $\lambda$ is a constant. We shall show a Ricci soliton by $(M, g, V, \lambda)$ the vector field $V$ of which is the potential vector field of the Ricci soliton.

Definition 1.7. A Hoph hypersurface $M$ in a Sasakian space form $\widetilde{M}^{2 n+1}(c)$ is said to be a locally symmetric hypersurface if it satisfies

$$
\left(\nabla_{W} R\right)(X, Y) Z=0
$$

for $X, Y, Z \in T M$, where $R$ and $\nabla$ denote the curvature tensor and Riemannian connection of $M$, respectively. 
Definition 1.8. Let $A$ be the shape operator of the hypersurface $M$ in $\widetilde{M}^{2 n+1}$ and the plane spanned by $\{\xi, U\}$ be invariant subspace of $A$. Then the hypersurface $M$ is called a Hopf hypersurface of $\widetilde{M}^{2 n+1}$.

Let $\widetilde{M}^{2 n+1}$ be a Sasakian manifold and let $M^{n}$ be a real submanifold of $\widetilde{M}^{2 n+1}$.

Definition 1.9. A CR-submanifold is a submanifold $M^{n}$ tangent to $\xi$ that admits an invariant distribution $D$ whose orthogonal complementary distribution $D^{\perp}$ is anti-invariant, that is, $T M=D \oplus D^{\perp}$ with condition $\varphi\left(D_{p}\right) \subset D_{p}$ for all $p \in M$ and $\varphi\left(D_{p}^{\perp}\right) \subset T_{p}^{\perp} M$ for all $p \in M$.

\subsection{Hopf Hypersurfaces in Sasakian space form}

Let $M$ be a Hoph hypersurface of a Sasakian space form $\widetilde{M}^{2 n+1}(c)$. We define a metric $g$ on $M$ by

$$
g(X, Y)=\widetilde{g}(\iota X, \iota Y)
$$

for any $X, Y \in T M$, where the Riemannian metric $g$ is said to be the induced metric from $\widetilde{g}$ and the $\iota$ is called an isometric immersion.

We shall need the Gauss and Weingarten formulas

$$
\widetilde{\nabla}_{X} Y=\nabla_{X} Y+h(X, Y), \quad \quad \widetilde{\nabla}_{X} N=-A X
$$

where $\widetilde{\nabla}$ and $\nabla$ are the Riemannian connection of $\widetilde{M}^{2 n+1}(c)$ and $M$, respectively, and $h$ is the second fundamental form, $N$ is a locally unit normal vector field on $M$, and $A$ is the shape operator with respect to $N$. The Gauss and Codazzi equations:

$$
\begin{aligned}
& R(X, Y) Z=\frac{c+3}{4}\{g(Y, Z) X-g(X, Z) Y\} \\
&-\frac{c-1}{4}\{\eta(Z)[\eta(Y) X-\eta(X) Y]+[g(Y, Z) \eta(X)-g(X, Z) \eta(Y)] \xi \\
&-g(\varphi Y, Z) \varphi X+g(\varphi X, Z) \varphi Y+2 g(\varphi X, Y) \varphi Z\} \\
&+g(A Y, Z) A X-g(A X, Z) A Y, \\
&\left(\nabla_{X} A\right) Y-\left(\nabla_{Y} A\right) X=\frac{c-1}{4}\{u(X) \varphi Y-u(Y) \varphi X-2 g(\varphi X, Y) U\} .
\end{aligned}
$$

Hence Ricci tensor is written as

$$
\begin{aligned}
\operatorname{Ric}(X, Y) & =\left\{\frac{(2 n+1) c+6 n-5}{4}\right\} g(X, Y)-\frac{(2 n+1) c-2 n-1}{4} \eta(X) \eta(Y) \\
& +(\operatorname{trace} A) g(A X, Y)-g(A X, A Y) .
\end{aligned}
$$

for $X, Y, Z \in T M$ and $u(X):=g(U, X)$, where $R$ and Ric are the curvature and Ricci tensors of $M$, respectively. 
Lemma 1.1. Let $M$ be a hypersurface of a Sasakian manifold $\widetilde{M}^{2 n+1}$. Then $A \xi=$ $-U$ such that $U$ is a vector field on $M$ with $\varphi U=N$.

Proof. By using the second relation (1.5) and the Gauss formula, we obtain

$$
0=-\varphi \xi=\widetilde{\nabla}_{\xi} \xi=\nabla_{\xi} \xi+g(A \xi, \xi) N .
$$

Thus, by separating the normal and tangential parts, we conclude

$$
\nabla_{\xi} \xi=0, \quad g(A \xi, \xi)=0 .
$$

Again, the second relation (1.5) and the Gauss formula imply

$$
-\varphi X=\widetilde{\nabla}_{X} \xi=\nabla_{X} \xi+g(A X, \xi) N
$$

and comparing the tangential part and the normal part, we get

$$
\nabla_{X} \xi=-\varphi X, \quad g(A X, \xi)=0 .
$$

Thus, from (1.10) and (1.11), it follows $A \xi=\delta U$ and the Gauss formula implies

$$
\nabla_{U} \xi+g(A U, \xi) N=\widetilde{\nabla}_{U} \xi=-\varphi U=-N
$$

then, $\nabla_{U} \xi=0$ and $g(A U, \xi)=-1$. Hence

$$
-1=g(A U, \xi)=g(U, A \xi)=\delta g(U, U)=\delta .
$$

therefore $\delta=-1, A \xi=-U$, which completes the proof.

Lemma 1.2. Let $M$ be a hypersurface of a Sasakian manifold $\widetilde{M}^{2 n+1}$. Then $A U=$ $-\xi+\beta U$

Proof. From the Gauss and Weingarten formula, it follows

$$
\varphi A X=-\widetilde{\nabla}_{X} \varphi N=\widetilde{\nabla}_{X} U=\nabla_{X} U+g(A X, U) N
$$

Comparing the vertical part and the horizontal part, we conclude

$$
\nabla_{X} U=\varphi A X, \quad g(A U, X)=0,
$$

then for any $X \in D$ we have $g(A U, X)=0$. So $A U=\alpha \xi+\beta U$.

Moreover, the Weingarten formula can be written as follows

$$
-N=\varphi A \xi=\widetilde{\nabla}_{\xi} U=\nabla_{\xi} U+g(A \xi, U) N
$$

then, we obtain

$$
\nabla_{\xi} U=0 \quad-1=g(A U, \xi)=\alpha g(\xi, \xi)+\beta g(U, \xi)=\alpha
$$

This shows that $\alpha=-1, A U=-\xi+\beta U$.

thus, by the lemmas (2.1) and (2.2), distribution $D^{\perp}$ is $A$ - invariant so distribution $D$ is $A$ - invariant, too, and $M$ is a Hoph hypersurface in the Sasakian manifold $\widetilde{M}^{2 n+1}$. 


\section{Ricci soliton on hypersurfaces with two distinct principal curvatures $k_{1}, k_{2}$}

We assume that $(M, g)$ is a Hoph hypersurface of a Sasakian space form $\widetilde{M}^{2 n+1}(c)$ with two distinct principal curvatures $k_{1}, k_{2}$. Also, let $\left\{e_{1}, \ldots, e_{n-1}, e_{n}=\varphi e_{1}, \ldots, e_{2 n-2}=\right.$ $\left.\varphi e_{n-1}, U, \xi\right\}$ be a local orthonormal frame field.

First, we consider a Hoph hypersurface $(M, g)$ of a Sasakian space form $\widetilde{M}^{2 n+1}(c)$ with $A X=k_{2} X$, for any vector field $X \in D$, and $A X=k_{1} X$, for any vector field $X \in D^{\perp}$.

Now, using the lemmas $(1.1)$ and (1.2) it follows that $\{U, \xi\}$ are not principal directions. To obtain the other principal curvatures, we consider eigenvectors $W_{1}, W_{2}$ with corresponding principal curvatures $\gamma_{1}, \gamma_{2}$, such that there must be linear combinations of $\{U, \xi\}$, and we put

$$
\begin{aligned}
& W_{1}=(\cos \theta) U+(\sin \theta) \xi \\
& W_{2}=(\cos \theta) \xi-(\sin \theta) U
\end{aligned}
$$

Then, the relations $A \xi=-U, A U=-\xi+\beta U$ imply

$$
\begin{aligned}
& \left(\gamma_{1} \cos \theta\right) U+\left(\gamma_{1} \sin \theta\right) \xi=-(\cos \theta) \xi+(\beta \cos \theta-\sin \theta) U \\
& \left(\gamma_{2} \cos \theta\right) \xi-\left(\gamma_{2} \sin \theta\right) U=(\sin \theta) \xi-(\cos \theta+\beta \sin \theta) U .
\end{aligned}
$$

As vector fields $\{U, \xi\}$ are linear independent, we get

$$
\gamma_{1} \sin \theta=-\cos \theta, \quad \gamma_{2} \cos \theta=\sin \theta
$$

and

$$
\begin{gathered}
-\sin \theta+\beta \cos \theta=\gamma_{1} \cos \theta \\
\cos \theta+\beta \sin \theta=\gamma_{2} \sin \theta
\end{gathered}
$$

From (2.2) and (2.3), it follows

$$
\gamma_{1}=\beta-\tan \theta \quad \gamma_{2}=\cot \theta+\beta .
$$

Relation(2.1) implies

$$
\gamma_{1}=-\cot \theta, \quad \gamma_{2}=\tan \theta
$$

Thus, from (2.4) and (2.5), we conclude

$$
\gamma_{1} \gamma_{2}=-1, \quad \gamma_{1}+\gamma_{2}=\beta
$$

On the other hand, by assumption for any vector field $X \in D^{\perp}, A X=k_{1} X$. That by the first relation $(2.6), k_{1}^{2}=-1$, and this is a contradiction. 
Theorem 2.1. There is not a Hoph hypersurface in a Sasakian space form $\widetilde{M}^{2 n+1}(c)$ with $A X=k_{2} X$, for any vector field $X \in D$ and $A X=k_{1} X$, for any vector field $X \in D^{\perp}$.

Then, we continue our study of Hohp hypersurfaces in a Sasakian space form and consider a Hoph hypersurface $(M, g)$ of a Sasakian space form $\widetilde{M}^{2 n+1}(c)$ such that for any vector field $X \in D, A X=k_{2} X$ and vector fields $\left\{W_{1}, W_{2}\right\} \in D^{\perp}$ are principal curvature vectors with the corresponding principal curvatures $k_{1}, k_{2}$ such that

$$
\begin{aligned}
& W_{1}=(\cos \theta) U+(\sin \theta) \xi \\
& W_{2}=(\cos \theta) \xi-(\sin \theta) U
\end{aligned}
$$

We compute

$$
k_{1} k_{2}=-1 \quad k_{1}+k_{2}=\beta
$$

Hence, by Relation (1.9), the Ricci tensor related to a Hoph hypersurface $(M, g)$ is written as

$(2 \mathbb{R}) c\left(e_{i}, e_{j}\right)=\left\{(2 n-2) k_{2}^{2}+\frac{(2 n+1) c+6 n-9}{4}\right\} \delta_{i j}, \quad(i, j=1, \ldots, 2 n-2)$,

$$
\begin{gathered}
\operatorname{Ric}(\xi, \xi)=2 n-2, \\
\operatorname{Ric}(U, U)=\left\{(2 n-2) k_{2}^{2}+\frac{(2 n+1) c-2 n-1}{4}\right\}, \\
\operatorname{Ric}(U, \xi)=-(2 n-2) k_{2}, \\
\operatorname{Ric}\left(e_{i}, \xi\right)=0, \quad(i=1, \ldots, 2 n-2), \\
\operatorname{Ric}\left(e_{i}, U\right)=0, \quad(i=1, \ldots, 2 n-2) .
\end{gathered}
$$

We consider a Hoph hypersurface $M(n>1)$ of a Sasakian space form $\widetilde{M}^{2 n+1}(c)$ satisfying the Ricci soliton equation

$$
\frac{1}{2} \mathcal{L}_{V} g+R i c-\lambda g=0
$$

with respect to the potential vector field $V$ on $M$ for constant $\lambda$. We suppose $M$ has two distinct principal curvatures $k_{1}, k_{2}$, with $m\left(k_{1}\right)=1, m\left(k_{2}\right)=2 n-1$. We put

$$
V:=f U, \quad(f: M \rightarrow \mathbb{R}, \quad f \neq 0)
$$


The definition of the Lie derivative and the first relation (1.12) imply

$$
\left(\mathcal{L}_{f U} g\right)(X, Y)=d f(X) u(Y)+d f(Y) u(X)+f\{g((\varphi A-A \varphi) X, Y)\} .
$$

We obtain

$$
\left(\mathcal{L}_{f U} g\right)(\xi, \xi)=0
$$

$$
\left(\mathcal{L}_{f U} g\right)(U, U)=2 d f(U)
$$$$
\left(\mathcal{L}_{f U} g\right)(U, \xi)=d f(\xi)
$$

From (2.8)-(2.13) and (2.16)-(2.21), the Ricci soliton equation (2.14) follows

$$
\left(\mathcal{L}_{f U} g\right)\left(\xi, e_{i}\right)=0, \quad(i=1, \ldots, 2 n-2),
$$

$$
\left(\mathcal{L}_{f U} g\right)\left(U, e_{i}\right)=d f\left(e_{i}\right), \quad(i=1, \ldots, 2 n-2),
$$

$$
\left(\mathcal{L}_{f U} g\right)\left(e_{i}, e_{j}\right)=0, \quad(i, j=1, \ldots, 2 n-2) .
$$

$$
\lambda=2 n-2,
$$

$$
d f(U)=(2-2 n) k_{2}^{2}-\frac{(2 n+1) c-10 n+7}{4},
$$

$$
d f(\xi)=(4 n-4) k_{2},
$$

$$
d f\left(e_{i}\right)=0, \quad(i=1, \ldots, 2 n-2),
$$

$$
\begin{aligned}
& \left(\frac{(2 n+1) c+6 n-5}{4}+(2 n-2) k_{2}^{2}+k_{1} k_{2}-\lambda\right) \delta_{i j}=0, \\
& (i, j=1, \ldots, 2 n-2) .
\end{aligned}
$$

By relations (2.22), (2.26) and the first relation (2.6), we have

$$
k_{2}^{2}=\frac{(2 n+1)(1-c)}{4(2 n-2)},
$$

Therefore, $k_{2}$ is constant. Thus, by the first relation $(2.6), k_{1}$ is constant.

Lemma 2.1. Let $M$ be a Hoph hypersurface with Ricci solitons $(M, g, f U, \lambda)$ of a Sasakian space form $\widetilde{M}^{2 n+1}(c)$. Then the principal curvatures $k_{1}, k_{2}$ on $D, D^{\perp}$ are constant. 
Also, by the second relation (2.6), $\beta$ is constant.

Theorem 2.2. A Ricci soliton $(M, g, V, \lambda)$ on a Hoph hypersurface $(M, g)$ with $A X=k_{2} X$ for any vector field $X \in D$ and $A W_{i}=k_{i} W_{i}$ for vector fields $\left\{W_{i}\right\} \in$ $D^{\perp}, i=\{1,2\}$ of a Sasakian space form $\widetilde{M}^{2 n+1}(c<1)$ with the potential vector field $V:=f U$ is a shrinking Ricci soliton with $\lambda=2 n-2$

Theorem 2.3. There is not a Hoph hypersurface with $A X=k_{2} X$ for any vector field $X \in D$ and $A W_{i}=k_{i} W_{i}$ for vector fields $\left\{W_{i}\right\} \in D^{\perp}, i=\{1,2\}$ of a Sasakian space form $\widetilde{M}^{2 n+1}(c \geq 1)$ such that $(M, g, V, \lambda)$ be a Ricci soliton with the potential vector field $V:=f U$.

Proof. If $c=1$, then by using $(2.27), k_{2}=0$ and this is a contradiction with $k_{1} k_{2}=-1$.

If $c>1$, then by (2.27), $k_{2}^{2}<0$ and this is a contradiction. Thus the proof is complete.

Finally, we consider a Hoph hypersurface $M(n>1)$ of a Sasakian space form $\widetilde{M}^{2 n+1}(c)$ satisfying the Ricci soliton equation with the potential vector field $V:=$ $f \xi$.

Using the definition of the Lie derivative and the second relation (1.5), it follows

$$
\left(\mathcal{L}_{f \xi} g\right)(X, Y)=d f(X) \eta(Y)+d f(Y) \eta(X) .
$$

We compute

$$
\left(\mathcal{L}_{f \xi} g\right)(\xi, \xi)=2 d f(\xi)
$$

$$
\left(\mathcal{L}_{f \xi} g\right)(U, U)=0
$$

$$
\left(\mathcal{L}_{f \xi} g\right)(U, \xi)=d f(U)
$$

By relations (2.8)-(2.13) and (2.28)-(2.33), the Ricci soliton equation (2.14) is equivalent to

$$
d f(\xi)=\lambda-2 n+2,
$$




$$
\begin{gathered}
\lambda=(2 n-2) k_{2}^{2}+\frac{(2 n+1) c-2 n-1}{4}, \\
d f(U)=(4 n-4) k_{2}, \\
d f\left(e_{i}\right)=0 \quad(i=1, \ldots, 2 n-2),
\end{gathered}
$$

$$
\left(\frac{(2 n+1) c+6 n-9}{4}+(2 n-2) k_{2}^{2}-\lambda\right) \delta_{i j}=0, \quad(i, j=1, \ldots, 2 n-2) .
$$

In the relation (2.38), if $i=j$, then

$$
\lambda=(2 n-2) k_{2}^{2}+\frac{(2 n+1) c+6 n-9}{4}
$$

Theorem 2.4. There is not any Hoph hypersurface $M$ with $A X=k_{2} X$ for any vector field $X \in D$ and $A W_{i}=k_{i} W_{i}$ for vector fields $\left\{W_{i}\right\} \in D^{\perp}, i=\{1,2\}$ of a Sasakian space form $\widetilde{M}^{2 n+1}(c)$ such that $(M, g, V, \lambda)$ be a Ricci soliton with potential vector field $V:=f \xi$.

Proof. by comparing (2.35) and (2.39), we obtain $n=1$ that this is a contradiction, which completes the proof.

\section{3. $\quad \eta$ - Ricci solitons on Hoph hypersurfaces in Sasakian space form}

Let $M$ be a Hoph hypersurface of a Sasakian space form $\widetilde{M}^{2 n+1}(c)$. Differentiating $A U=-\xi+\beta U$ covariantly, we obtain

$$
\left(\nabla_{X} A\right) U=\varphi X-A \varphi A X+\beta \varphi A X+(X \beta) U
$$

From the Codazzi equation, it follows

$$
\begin{aligned}
\left(\nabla_{U} A\right) X & =\frac{c+3}{4}\{\varphi X\}-A \varphi A X+\beta \varphi A X+(X \beta) U \\
& -\frac{c-1}{4} u(X) N
\end{aligned}
$$

By comparing the tangential part and the normal part in the relation above, we conclude

$$
\left(\nabla_{U} A\right) X=\frac{c+3}{4}\{\varphi X\}-A \varphi A X+\beta \varphi A X+(X \beta) U
$$

and

$$
U \perp X \quad \text { or } \quad c=1
$$


While $c=1$, then the ambient Sasakian space form is a sphere $S^{2 n+1}$. since $\nabla_{U} A$ is self-adjoint and therefore

$$
\begin{aligned}
0 & =-2 g(A \varphi A X, Y)+\frac{c+3}{2} g(\varphi X, Y)+\beta g((A \varphi+\varphi A) X, Y) \\
& +(X \beta) u(Y)-(Y \beta) u(X)
\end{aligned}
$$

Substituting $Y$ for $U$ in (3.3), we obtain $(X \beta)=g(U, X) U \beta$. Similarly by substituting $X$ for $U$ in (3.3), we obtain $(Y \beta)=g(U, Y) U \beta$.

It follows

$$
2 A \varphi A X-\frac{c+3}{2} \varphi X=\beta(A \varphi+\varphi A) X
$$

We assume that $A X=k X$ for any vector field $X \in D,\|X\|=1$. Then it follows that

$$
(2 k-\beta) A \varphi X=\left(k \beta+\frac{c+3}{2}\right) \varphi X .
$$

The case $2 k \neq \beta$ yields

$$
A \varphi X=\frac{\left(k \beta+\frac{c+3}{2}\right)}{(2 k-\beta)} \varphi X .
$$

We consider an $\eta$-Ricci soliton

$$
\frac{1}{2} \mathcal{L}_{U} g+R i c-\lambda g-\mu \eta \otimes \eta=0
$$

on a Hoph hypersurface $M(n>1)$ in a Sasakian space form $\widetilde{M}^{2 n+1}(c)$ where $U$ is the potential vector field on $M$ and $\lambda, \mu$ are constants. The first relation (1.12) implies

$$
\left(\mathcal{L}_{U} g\right)(X, Y)=g((\varphi A-A \varphi) X, Y) .
$$

By substituting (1.9) and (3.8) in the relation (3.7), it follows

$$
\begin{aligned}
(\text { trace } A) g(A X, Y) & -g\left(A^{2} X, Y\right)+\frac{1}{2} g((\varphi A-A \varphi) X, Y) \\
& +\left\{\frac{(2 n+1) c+6 n-5}{4}-\lambda\right\} g(X, Y) \\
& =\left\{\mu+\frac{(2 n+1) c-2 n-1}{4}\right\} \eta(X) \eta(Y) .
\end{aligned}
$$

Putting $X=U$, we may write

$$
\left\{(2 n-2)+\beta(\operatorname{trace} A)-\beta^{2}-\lambda\right\} U+\{\beta-\operatorname{trace} A\} \xi=0
$$

Since vector fields $\{U, \xi\}$ are linearly independent, we have

$$
\beta=\operatorname{trace} A, \quad \lambda=2 n-2 .
$$


using the relation (3.6) and the first relation (3.10), we derive

$$
k^{2}=-1
$$

Thus we prove the following lemma and theorem.

Lemma 3.1. Let $M$ be a Hoph hypersurface of a Sasakian space form $\widetilde{M}^{2 n+1}(c)$. If $X \in D$ is a principal direction then $\varphi X$ is a principal direction.

Theorem 3.1. If $M$ is a Hoph hypersurface of a Sasakian space form $\widetilde{M}^{2 n+1}(c)$ with $c=1$ then a Hoph hypersurface $M$ does not admit a $\eta$ - Ricci soliton with a potential vector field $U$.

Now, putting $X=\xi$ in the relation (3.9) we obtain

$$
\{2 n-2-\mu-\lambda\} \xi+\{\beta-\operatorname{trace} A\} U=0 .
$$

Since vector fields $\{U, \xi\}$ are linearly independent, we have

$$
\beta=\operatorname{trace} A, \quad \lambda+\mu=2 n-2 .
$$

If $\mu=0$, then $\lambda=2 n-2$. Therefore, using the relation (3.6) and the first relation (3.12), it follows

$$
k^{2}=-\frac{c+3}{4},
$$

which leads to

Lemma 3.2. Let $M$ be a Hoph hypersurface of a Sasakian space form $\widetilde{M}^{2 n+1}(c)$ with $c=-3$. Then $A=0$ on $D$.

Proof. By the relations (3.13) and (3.6), the proof is trivial.

Theorem 3.2. A Ricci soliton $(M, g, U, \lambda)$ on a Hoph hypersurface $(M, g)$ of a Sasakian space form $\widetilde{M}^{2 n+1}(c<-3)$ with a potential vector field $U$ is a shrinking Ricci soliton with $\lambda=2 n-2$

Theorem 3.3. There is not a Hoph hypersurface of a Sasakian space form $\widetilde{M}^{2 n+1}(c>$ -3) such that $(M, g, U, \lambda)$ is a Ricci soliton.

Proof. By the relations (3.13), the proof is trivial. 


\section{Ricci soliton on Locally symmetric hypersurfaces}

A Hoph hypersurface $M$ in a Sasakian space form $\widetilde{M}^{2 n+1}(c)$ is said to be a locally symmetric hypersurface if it satisfies

$$
\left(\nabla_{W} R\right)(X, Y) Z=0
$$

for any $X, Y, Z \in T M$.

We begin with several results on locally symmetric hypersurfaces of a Sasakian space form.

Lemma 4.1. Let $M$ be a locally symmetric hypersurface of a Sasakian space form $\widetilde{M}^{2 n+1}(c)$. Then $A \varphi=\varphi A$, for all $W \in D$

Proof. Putting $Y, Z=\xi$ in (4.1) and for any $X, W \in D$, we get

$$
\begin{aligned}
0 & =\left(\nabla_{W} R\right)(X, \xi) \xi \\
& =-g\left(\left(\nabla_{W} A\right) X, \xi\right) A \xi=g\left(\left(\nabla_{W} A\right) X, \xi\right) U \\
& =g\left(\nabla_{W} A X-A \nabla_{W} X, \xi\right) U \\
& =\left\{-g\left(A X, \nabla_{W} \xi\right)+g\left(\nabla_{W} X, U\right)\right\} U \\
& =\left\{-g\left(X, A \widetilde{\nabla}_{W} \xi\right)-g\left(X, \nabla_{W} U\right)\right\} U \\
& =\{g(X, A \varphi W)-g(X, \varphi A W)\} U
\end{aligned}
$$

therefore $A \varphi=\varphi A$.

Thus, if $X \in D$ is an eigenvector field corresponding to the principal curvature $k$, then $\varphi X$ is an eigenvector field corresponding to the principal curvature $k$. Thus $D$ is $A$ invariant.

Now, we denote the eigenvalue of all vector field on $D$ as equal, that is, if $X, Y$ are two eigenvector fields with eigenvalues $\nu, \omega$, respectively, then $\nu=\omega$.

Lemma 4.2. Let $M$ be a locally symmetric hypersurface of a Sasakian manifold $\widetilde{M}^{2 n+1}(c)$. If $X, Y$ are two eigenvectors field with eigenvalues $\nu, \omega$ on $D$, respectively, then $\nu=\omega$.

Proof. Let $X, Y \in D$ are principal curvature vectors with corresponding principal curvatures $\nu, \omega$. The first relation (1.12), $A \varphi=\varphi A$ and

$$
\begin{aligned}
g\left(\left(\nabla_{\xi} A\right) \xi, Y\right) & =g\left(\nabla_{\xi} A \xi, Y\right)-g\left(\left(\nabla_{\xi} \xi, A Y\right)\right. \\
& =-g\left(\nabla_{\xi} U, Y\right)=-g(\varphi A \xi, Y)=g(\varphi U, Y)=0 \\
\left(\nabla_{\xi} A\right) \xi & =\nabla_{\xi} A \xi-A \nabla_{\xi} \xi \\
& =-\nabla_{\xi} U=0 .
\end{aligned}
$$


imply

$$
\begin{aligned}
\left(\nabla_{\xi} R\right)(X, \xi) Y & =g\left(\left(\nabla_{\xi} A\right) \xi, Y\right) A X \\
& -g\left(\left(\nabla_{\xi} A\right) X, Y\right) A \xi-g(A X, Y)\left(\nabla_{\xi} A\right) \xi \\
& =(\nu-\omega) g(\varphi X, Y) U .
\end{aligned}
$$

Therefore, we conclude $\nu=\omega$.

Thus, for all vector fields $X \in D$, we have $A X=k X$. Let $(M, g)$ be a locally symmetric hypersurface and let $\left\{e_{1}, \ldots, e_{n-1}, e_{n}=\varphi e_{1}, \ldots, e_{2 n-2}=\varphi e_{n-1}, U, \xi\right\}$ be a local orthonormal frame field. Hence Ricci tensor is written as

$$
\operatorname{Ric}\left(e_{i}, e_{j}\right)=\left\{\frac{(2 n+1) c+6 n-5}{4}+k \beta+(2 n-3) k^{2}\right\} \delta_{i j},
$$

$$
\operatorname{Ric}(\xi, \xi)=2 n-2,
$$

$$
\begin{gathered}
\operatorname{Ric}(U, U)=\frac{(2 n+1) c+6 n-9}{4}+(2 n-2) k \beta, \\
\operatorname{Ric}(\xi, U)=(2-2 n) k,
\end{gathered}
$$

$$
\operatorname{Ric}\left(e_{i}, \xi\right)=0,
$$

$$
\operatorname{Ric}\left(e_{i}, U\right)=0
$$

Suppose that a locally symmetric hypersurface $M(n>1)$ of a Sasakian space form $\widetilde{M}^{2 n+1}(c)$ admits a Ricci soliton.

We put

$$
V:=f U, \quad(f: M \rightarrow \mathbb{R}, \quad f \neq 0) .
$$

The definition of the Lie derivative and the first relation (1.12) imply

(4.9) $\left(\mathcal{L}_{f U} g\right)(X, Y)=d f(X) u(Y)+d f(Y) u(X)+f\{g((\varphi A-A \varphi) X, Y)\}$.

By (4.9), we obtain

$$
\left(\mathcal{L}_{f U} g\right)(\xi, \xi)=0
$$

$$
\left(\mathcal{L}_{f U} g\right)(U, U)=2 d f(U)
$$

$$
\left(\mathcal{L}_{f U} g\right)(U, \xi)=d f(\xi)
$$




$$
\begin{gathered}
\left(\mathcal{L}_{f U} g\right)\left(\xi, e_{i}\right)=0, \quad(i=1, \ldots, 2 n-2), \\
\left(\mathcal{L}_{f U} g\right)\left(U, e_{i}\right)=d f\left(e_{i}\right), \quad(i=1, \ldots, 2 n-2), \\
\left(\mathcal{L}_{f U} g\right)\left(e_{i}, e_{j}\right)=0, \quad(i, j=1, \ldots, 2 n-2) .
\end{gathered}
$$

From the Codazzi equation, it follows

$$
\frac{c-1}{2} g\left(e_{i}, \varphi e_{j}\right)=g\left(\left(\nabla_{e_{i}} A\right) e_{j}-\left(\nabla_{e_{j}} A\right) e_{i}, U\right)
$$

By the relation above and Lemma (2.2), we obtain

$$
\left(k^{2}-k \beta-\frac{c+3}{4}\right) g\left(e_{i}, \varphi e_{j}\right)=0,
$$

if $i=n+j-1$, then $g\left(e_{i}, \varphi e_{j}\right)=1$ and therefore

$$
k \beta=k^{2}-\frac{c+3}{4} .
$$

By relations (4.3)-(4.8) and (4.10)-(4.15), the Ricci soliton equation(2.14) is equivalent to

$$
\begin{gathered}
\lambda=2 n-2, \\
d f(U)=(2-2 n) k^{2}-\frac{3 c-8 n+5}{4}, \\
d f(\xi)=(4 n-4) k, \\
d f\left(e_{i}\right)=0, \quad(i=1, \ldots, 2 n-2), \\
\left(\frac{(2 n+1) c+6 n-5}{4}+(2 n-3) k^{2}+k \beta-\lambda\right) \delta_{i j}=0,(i, j=1, \ldots, 2 n-2) .
\end{gathered}
$$

By the relations (4.17) and (4.22) and the relation (4.16), we conclude

$$
k^{2}=\frac{n(1-c)}{2(2 n-2)} .
$$

Lemma 4.3. Let $M$ be a locally symmetric hypersurface with the Ricci soliton $(M, g, f U, \lambda)$ in a Sasakian space form $\widetilde{M}^{2 n+1}(c)$. Then the eigenvalue $k$ on $D$ is constant.

Proof. By the relation (4.22), the proof is trivial. 
Also, from the above lemma and the relation (4.16), $\beta$ is constant.

Theorem 4.1. A Ricci soliton $(M, g, V, \lambda)$ on a locally symmetric hypersurface $(M, g)$ of a Sasakian space form $\widetilde{M}^{2 n+1}(c<1)$ with a potential vector field $V:=f U$ is a shrinking Ricci soliton with $\lambda=2 n-2$

Theorem 4.2. There is not a locally symmetric hypersurface $(M, g)$ of a Sasakian space form $\widetilde{M}^{2 n+1}(c \geq 1)$ such that $(M, g, V, \lambda)$ is a Ricci soliton with a potential vector field $V:=f U$.

Proof. By the relation (4.22), the proof is trivial.

\section{REF E R E N C ES}

1. C. Boyer And K. GAlicki, Sasakian geometry, Oxford Mathematical Monographs. Oxford University Press, Oxford, 2008.

2. B.Y. CHEN, Ricci solitons on Riemannian submanifolds,Riemannian Geometry and Applications to Engineering and Economics Bucharest, Romania, Proceedings of the Conference RIGA 2014 (May 19-21, 2014, Bucurexsti).

3. J. T. Cho And M. KimurA, Ricci solitons and real hypersurfaces in a complex space form, Tohoku Math. J. 61 (2009), 205-212.

4. J. T. Сhо AND M. Kimura, Ricci solitons on locally conformally flat hypersurfaces in space forms, Journal of Geometry and Physics 62 (2012) 1882-1891.

5. B. Chow And D. Knopf, The Ricci flow: An introduction, Math. Surveys Monogr. 110, American Mathematical Society, Providence, RI, 2004.

6. R. S. Hamilton, The Ricci flow on surfaces Mathematics and general relativity ,(Santa Cruz, CA, 1986), Contemp. Math. 71, American Math. Soc., Providence, RI, (1988) 237-262.

7. C. HE AND M. ZHU, Ricci solitons on Sasakian manifolds,arXiv:1103.4407v2 [math.DG], 26 sep (2011).

8. T. IVEY, Ricci solitons on compact 3-manifolds, Differential Geom. Appl. 3 (1993), 301-307.

9. U.H. Ki, Real hypersurfaces with parallel Ricci tensor of a complex space form, Tsukuba J. Math. 13 (1989), 73-81.

10. U.K. KIM, Nonexistence of Ricci-parallel real hypersurfaces in $\mathrm{P}_{2} \mathrm{C}$ or $\mathrm{H}_{2} \mathrm{C}$, Bull. Korean Math. Soc. 41 (2004), 699-708.

11. J. Morgan And G. Tian, Ricci Flow and the Poincare Conjecture, Clay Mathematics Monographs, 5, Cambridge, MA, 2014.

12. G. Perelman, The entropy formula for the Ricci flow and its geometric applications, http://arXiv.org/ abs/math.DG/02111159, preprint. 
Esmail Abedi

Department of Mathematics

Azarbaijan Shahid Madani University

Tabriz 53751 71379, Iran

esabedi@azaruniv.edu

\section{Zahra Nazari}

Department of Mathematics Azarbaijan Shahid Madani University

Tabriz 53751 71379, Iran

z.nazari@azaruniv.edu 